\title{
Diagnostic Accuracy of Amino Acid and FDG-PET in Differentiating Brain Metastasis Recurrence from Radionecrosis after Radiotherapy: A Systematic Review and Meta-Analysis
}

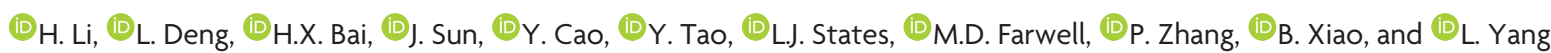

\begin{abstract}
BACKGROUND: Current studies that analyze the usefulness of amino acid and FDG-PET in distinguishing brain metastasis recurrence and radionecrosis after radiation therapy are limited by small cohort size.
\end{abstract}

PURPOSE: Our aim was to assess the diagnostic accuracy of amino acid and FDG-PET in differentiating brain metastasis recurrence from radionecrosis after radiation therapy.

DATA SOURCES: Studies were retrieved from PubMed, Embase, and the Cochrane Library.

STUDY SELECTION: Fifteen studies were included from the literature. Each study used PET to differentiate radiation necrosis from tumor recurrence in contrast-enhancing lesions on follow-up brain MR imaging after treating brain metastasis with radiation therapy.

DATA ANALYSIS: Data were analyzed with a bivariate random-effects model. Sensitivity, specificity, positive likelihood ratio, negative likelihood ratio, and diagnostic odds ratio were pooled, and a summary receiver operating characteristic curve was fit to the data.

DATA SYNTHESIS: The overall pooled sensitivity, specificity, positive likelihood ratio, negative likelihood ratio, and diagnostic odds ratio of PET were $0.85,0.88,7.0,0.17$, and 40 , respectively. The area under the receiver operating characteristic curve was 0.93 . On subgroup analysis of different tracers, amino acid and FDG-PET had similar diagnostic accuracy. Meta-regression analysis demonstrated that the method of quantification based on patient, lesion, or PET scan (based on lesion versus not, $P=.07$ ) contributed to the heterogeneity.

LIMITATIONS: Our study was limited by small sample size, and $60 \%$ of the included studies were of retrospective design.

CONCLUSIONS: Amino acid and FDG-PET had good diagnostic accuracy in differentiating brain metastasis recurrence from radionecrosis after radiation therapy.

ABBREVIATIONS: SRS = stereotactic radiosurgery; QUADAS = Quality Assessment of Diagnostic Accuracy Studies; "C-MET = ["C]-methyl-L-methionine; ${ }^{18} \mathrm{~F}$-FET $=\mathrm{O}-\left(2-\left[{ }^{18} \mathrm{~F}\right]\right.$ fluoroethyl)-L-tyrosine; ${ }^{18} \mathrm{~F}-\mathrm{FDOPA}=\mathrm{L}-3,4-$ dihydroxy $-6-\left[{ }^{18} \mathrm{~F}\right]$-fluorophenylalanine

etastatic brain tumors are the most common brain tumor in adults, with incidence estimates ranging from 200,000 to 300,000 patients per year. ${ }^{1}$ The frequency of brain metastasis is

Received June 14, 2017; accepted after revision September 19

From the Department of Neurology (H.L., L.D., L.Y.), Second Xiangya Hospital of Central South University, Changsha, Hunan Province, People's Republic of China; Departments of Radiology (H.X.B., J.S., M.D.F.) and Pathology (P.Z.), Hospital of the University of Pennsylvania, Philadelphia, Pennsylvania; Cancer Research Institute (Y.C., Y.T.), Central South University, Changsha, Hunan Province, People's Republic of China; Department of Radiology (L.J.S.), Children's Hospital of Philadelphia, Philadelphia, Pennsylvania; and Department of Neurology (B.X.), Xiangya Hospital of Central South University, Changsha, Hunan Province, People's Republic of China. This study was supported by Shenghua Yuying Project of Central South University to Li Yang.

Please address correspondence to Li Yang, MD, Department of Neurology, Second Xiangya Hospital, Central South University, No. 139 Middle Renmin Rd, Changsha, Hunan Province, 410011, People's Republic of China; e-mail: yangli762@csu.edu.cn and Bo Xiao, MD, Department of Neurology, Xiangya Hospital, Central South increasing, likely due to increased frequency of advanced imaging procedures performed. ${ }^{2}$ Prognosis can vary widely and depends on age, performance status, number of lesions, extracranial disease status, and primary malignancy. ${ }^{3}$ More recently, radiation therapy, particularly stereotactic radiosurgery (SRS), has become an increasingly important treatment option for the initial management of patients with brain metastasis. ${ }^{4}$ The efficacy of SRS, when used alone or combined with whole-brain radiation therapy, has been demonstrated in Phase III studies and has shown a 12 -month local control rate of 70\%-90\%. . $^{5-8}$

One of the most common complications after SRS for brain metastasis is radiation injury. These injuries can either be reversible,

University, No. 87 Xiangya Rd, Changsha, Hunan Province, 410000, People's Republic of China; e-mail: xiaobo_xy@126.com

三 Indicates article with supplemental on-line tables.

http://dx.doi.org/10.3174/ajnr.A5472 
such as swelling of the tumor or demyelination, or irreversible, such as liquefactive or coagulative necrosis. ${ }^{9,10}$ The irreversible injury, also called radionecrosis, can occur during the same period as tumor recurrence. ${ }^{11}$ It occurs in approximately $25 \%$ of patients after radiation therapy. ${ }^{12,13}$ While conventional MR imaging remains the most accurate technique for detecting the presence of brain metastasis, the appearance of metastasis is similar to that of radionecrosis on contrast-enhanced T1-weighted and T2-weighted sequences. ${ }^{14-16}$ As a consequence, it is often impossible to distinguish radiation necrosis from tumor relapse either clinically or with MR imaging. In current practice, many imaging methods such as advanced MR imaging (eg, dynamic susceptibility contrast perfusion and susceptibilityweighted imaging, diffusion-weighted imaging), MRS, perfusionweighted MR imaging, SPECT, and PET have been used empirically to address this problem. ${ }^{17}$

New postprocessing techniques such as textural analysis and MR fingerprinting hold great promise for differentiating radiation necrosis from tumor recurrence. ${ }^{18-20}$ However, no relevant studies have been performed using these techniques because they are difficult to implement and data training requires a large number of patients, which are difficult to recruit from a single institution. Of these imaging methods, metabolic PET imaging is the most widely studied due to its availability and established clinical utility for other indications. 2- $\left[{ }^{18} \mathrm{~F}\right]$ fluoro-2-deoxy-D-glucose $\left({ }^{18} \mathrm{~F}-\mathrm{FDG}\right)$ was used as the PET radiotracer in some early studies, but the high physiologic glucose consumption of the brain and the variable glucose uptake of metastatic brain lesions limited its use. ${ }^{21,22}$ Recently, amino acid PET imaging using $\left[{ }^{11} \mathrm{C}\right]$-methyl-L-methionine $\left({ }^{11} \mathrm{C}-\mathrm{MET}\right)$, O-(2$\left[{ }^{18} \mathrm{~F}\right]$ fluoroethyl)-L-tyrosine $\left({ }^{18} \mathrm{~F}-\mathrm{FET}\right)$, and L-3,4-dihydroxy-6$\left[{ }^{18} \mathrm{~F}\right]$-fluorophenylalanine $\left({ }^{18} \mathrm{~F}-\mathrm{FDOPA}\right)$ have demonstrated more promising results. ${ }^{8,23,24}$

However, current studies that analyze the usefulness of amino acid and FDG-PET in distinguishing brain metastasis recurrence and radionecrosis after radiation therapy are limited by a small cohort size. Thus, we conducted a meta-analysis to assess the diagnostic accuracy of amino acid and FDG-PET in differentiating brain metastasis recurrence from radionecrosis after radiation therapy.

\section{MATERIALS AND METHODS Search Strategy}

We searched PubMed (1966-2017), Embase (1980-2017), and the Cochrane Library (1996-2017). The studies chosen were restricted to humans, but not restricted by date, language, or publication status. We used the following combined search terms: (Positron-Emission Tomography, PET) AND (recurrence, recurrence ${ }^{\star}$, recurrent, relapse $^{\star}$, recrudescence ${ }^{\star}$, neoplasm metastasis, metastas ${ }^{\star}$, progression) AND (radionecrosis, radiation necrosis, radiation-induced necrosis, posttreatment necrosis, radiation injury, radionecrotic, postradiotherapy necrosis) AND (radiation therapy, radiotherap ${ }^{*}$, radiation therap ${ }^{\star}$, radiation treatment, radiosurgery, radiosurger ${ }^{\star}$ ). We combined the terms appropriately with Medical Subject Headings terms and used an appropriate adjustment for different data bases. Details of the search strategies can be found in the Appendix.

\section{Selection Criteria}

Studies were selected if they included the following: 1) subjects who underwent amino acid or FDG-PET for differentiating brain metas- tasis recurrence from radionecrosis after radiation therapy; 2) follow-up data that enabled the building of a $2 \times 2$ contingency table to calculate sensitivity and specificity; and 3 ) at least 10 subjects who underwent both the experimental test (PET) and the reference standard test (histopathology or clinical and radiologic follow-up) for the final brain metastasis recurrence or radionecrosis diagnosis.

We excluded the following types of studies: 1) letters, case reports, editorials, review articles, and animal studies; 2) nonoriginal and unpublished studies; 3 ) those that used a patient cohort that overlapped the cohort of a previous study; and 4) those that included no detailed information about the diagnostic and quantitative accuracy of PET.

\section{Data Extraction}

After literature selection, studies in full text were reviewed by 2 authors (H.L. and L.D.). We retrieved data including first author, publication year, country, study design, the number of patients, the number of lesions, the method of quantification, age, sex, primary tumor, follow-up time, radiation therapy methods, tracer, standard references, and cutoff index. The number of truepositive, false-positive, false-negative, and true-negative was calculated. The method of quantification could be based on lesion, patient, or PET scan. If a study did not specify how many lesions each patient had and used each patient as the unit of measurement in reporting their results, the numbers were counted on the basis of the patient. If a study contained lesions from different PET scans of the same patient (eg, after repeat treatment), these lesions were counted separately and the method of quantification was defined on the basis of the PET scan. All discrepancies were resolved in consensus after discussion.

\section{Quality Assessment}

Two authors (H.L. and L.D.) assessed the methodologic quality of the selected studies by using Quality Assessment of Diagnostic Accuracy Studies (QUADAS)-2 (http://annals.org/aim/article/ 474994/quadas-2-revised-tool-quality-assessment-diagnosticaccuracy-studies). ${ }^{25}$ Discrepancies were resolved in consensus meetings by a panel including a third author (H.X.B.). The risk of bias was analyzed in 4 domains: patient selection, index test, reference standard, and flow and timing. Applicability was assessed in the following 3 domains: patient selection, index test, and reference standard. Signaling questions were applied to determine the risk of bias and applicability.

\section{Statistical Analysis}

We used bivariate random-effects models described previously by Reitsma et $\mathrm{al}^{26}$ to perform the meta-analysis. The pooled estimates of sensitivity, specificity, positive likelihood ratio, negative likelihood ratio, and diagnostic odds ratios and their corresponding 95\% confidence intervals were calculated, and forest plots were drawn. In addition, a summary receiver operating characteristic curve was fit to the estimates of sensitivity, specificity, and corresponding variances, and the area under the receiver operating characteristic curve was calculated. The summary receiver operating characteristic curve and the area under the receiver operating characteristic curve were used to provide an overall summary of the test performance. This study considered that an area under the receiver operating characteristic curve of $>0.90$ indi- 

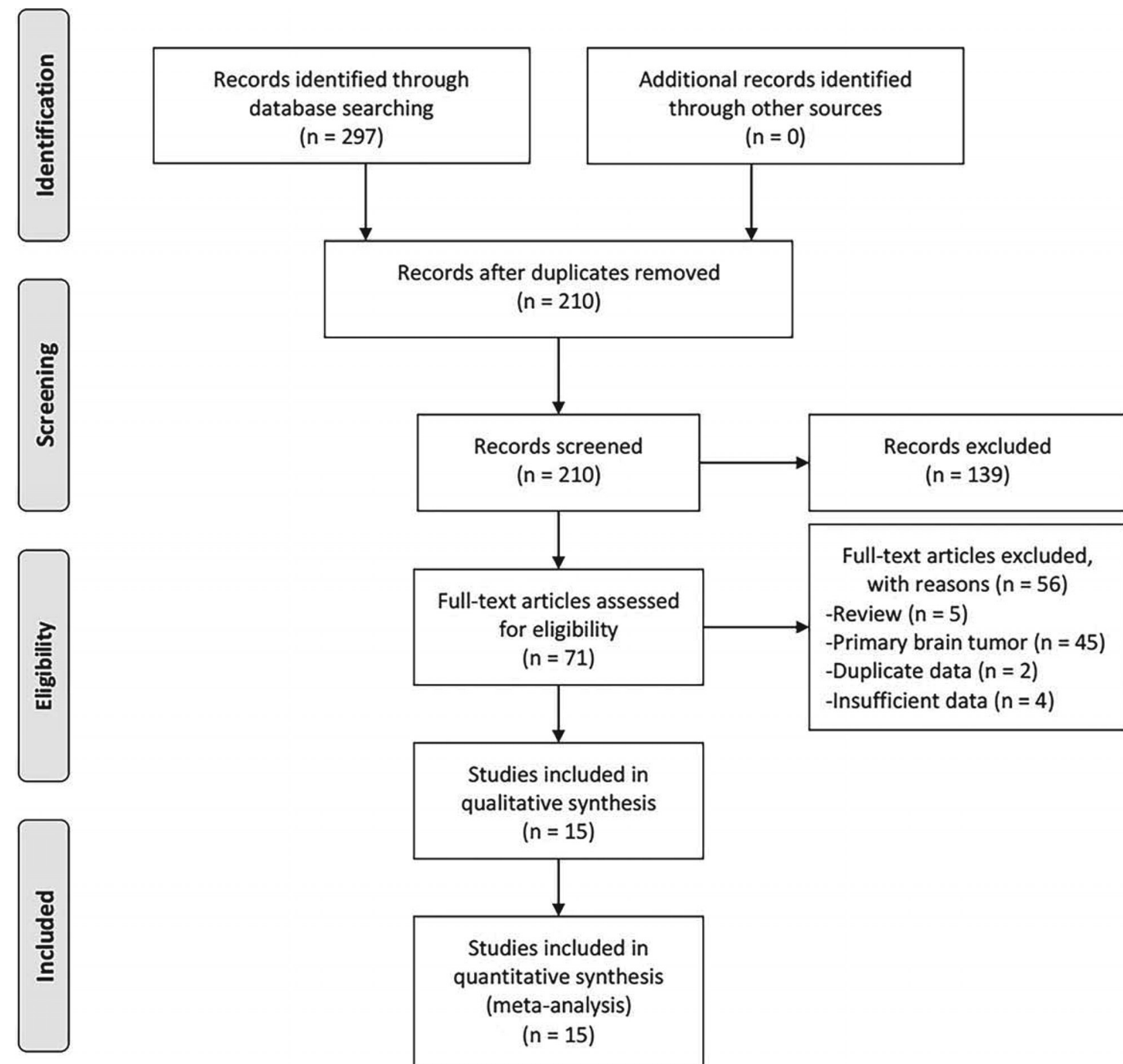

Studies included in

qualitative synthesis

$(n=15)$

FIG 1. Preferred Reporting Items for Systematic Reviews and Meta-Analyses (PRISMA; http://prisma-statement.org/) 2009 flow diagram.

cated high accuracy and an area under the receiver operating characteristic curve near 0.50 indicated low accuracy. ${ }^{27}$ Interstudy heterogeneity was assessed by calculating the $\mathrm{I}^{2}$ and Cochran Q statistics for each forest plot, and the difference was considered statistically significant when the $P$ value was $<.05$. The $\mathrm{I}^{2}$ values were used to determine the proportion of the variation among the studies attributable to heterogeneity rather than chance. Heterogeneity was ranked low (25\%), moderate $(50 \%)$, and high $(75 \%) .{ }^{28}$ The posttest probability of tumor recurrence was computed on the basis of likelihood ratios, and pretest probability, by applying the Bayes theorem. ${ }^{29,30}$

Additionally, sensitivity analysis was conducted using the leave-one-out approach by excluding studies one by one to evaluate the influences of individual studies on the final effects. Univariate meta-regression and subgroup analysis were used to explore the sources of heterogeneity. The covariates investigated included study design, country, radiation therapy methods, tracer, cutoff index, analytic method used, sample size, median age, and male percentage. Finally, publication bias was evaluated by the Deeks test for funnel plot asymmetry. All analyses were performed by using STATA IC 14 (StataCorp, College Station, Texas).

\section{RESULTS}

\section{Literature Search and Characteristics of the Included \\ Studies}

A total of 297 studies were identified, of which 87 were excluded because of duplication. After we read the titles and abstracts, a further 139 studies were excluded. The remaining 71 full-text studies were carefully reviewed, and we eliminated the following: review articles $(n=5)$, primary brain tumor $(n=45)$, duplicate data $(n=2)$, and insufficient data $(n=4)$. Finally, 15 studies $^{8,13,22-24,31-40}$ were included for further analysis (Fig 1). 


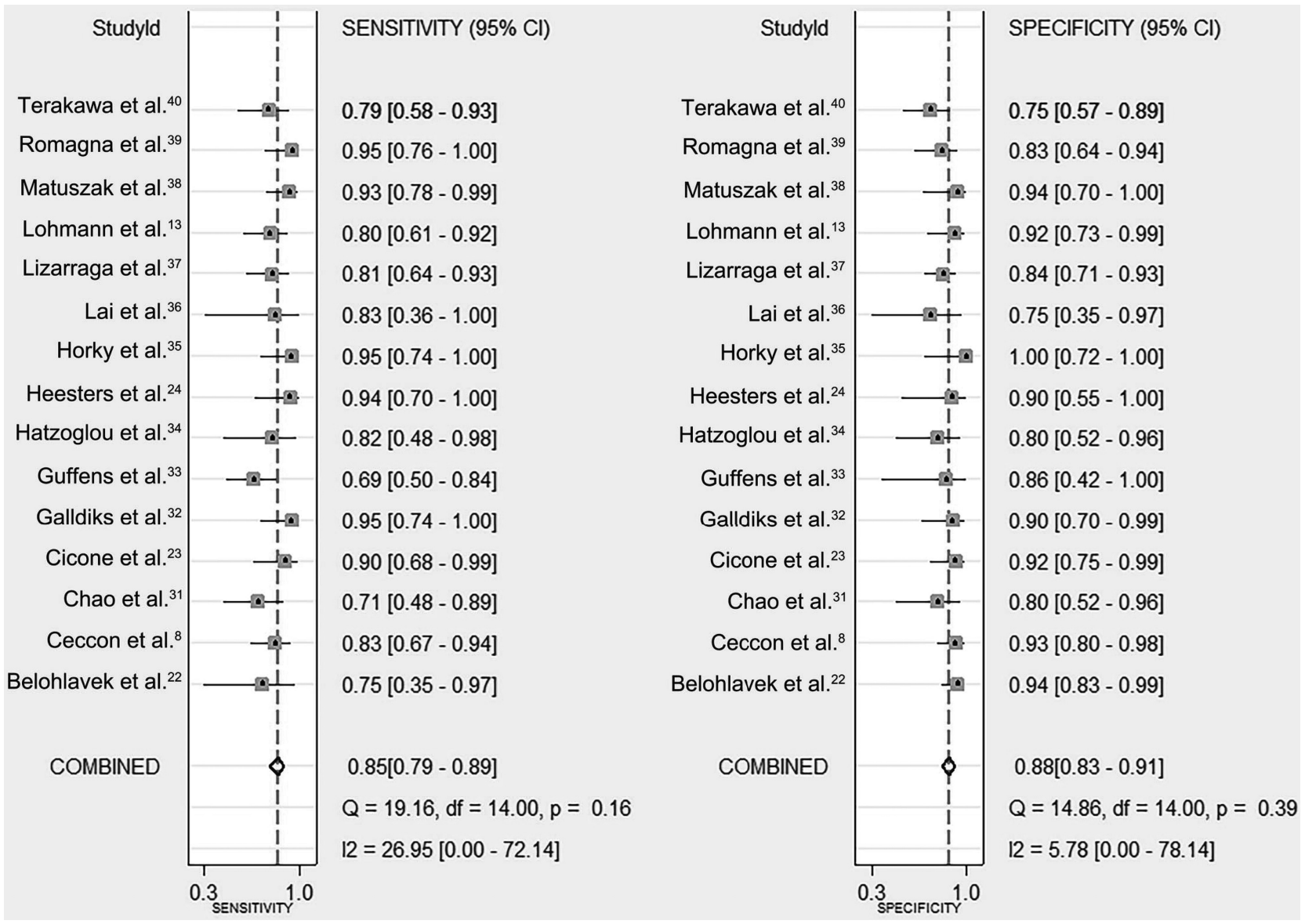

FIG 2. Pooled sensitivity and specificity of PET. The point estimates shown.

The characteristics of these studies are summarized in On-line Table 1 . The 15 studies encompassing 505 patients were published between 2001 and 2017. Six $^{22,23,32,34,36,39}$ were prospective, and $9^{8,13,24,31,33,35,37,38,40}$ were retrospective. The age of patients ranged from 17 to 81 years. Five studies ${ }^{31,34-37}$ were conducted in the United States; and $4,^{8,13,32,39}$ in Germany. The rest of the studies were conducted in other countries including the Czech Republic, ${ }^{22}$ Italy, ${ }^{23}$ Belgium, ${ }^{33}$ the Netherlands, ${ }^{24}$ France, ${ }^{38}$ and Japan. ${ }^{40}$ The primary tumors for the metastases were mainly from renal cell carcinoma, lung cancer, breast cancer, colorectal cancer, and melanoma. The radiation therapy methods were all SRS with or without other methods. Six studies ${ }^{22,31,34-36,38}$ used ${ }^{18} \mathrm{~F}-\mathrm{FDG}$ as the tracer, 5 studies ${ }^{8,13,32,33,39}$ used ${ }^{18}$ F-FET, 2 studies ${ }^{24,40}$ used ${ }^{11} \mathrm{C}$-MET, and 2 studies ${ }^{23,37}$ used ${ }^{18} \mathrm{~F}$-FDOPA. The standard reference used for all studies except Lai et $\mathrm{al}^{36}$ (only histopathology) was histopathology with clinical and/or radiologic follow-up.

\section{Quality Assessment}

Seven domains were assessed regarding bias and applicability for each article. ${ }^{25}$ Of the total 105 domains from all articles, 18 domains were determined as high risk ( 17 for risk of bias and 1 for applicability concerns). Two domains were determined to be of unclear risk because of the incomplete reporting of the studies. Therefore, the overall quality was acceptable. We found that bias stemmed mainly from the patient selection $(n=9)$, index test (high risk, $n=5$; unclear risk, $n=2)$, and flow and timing $(n=2)$ domains. The quality assessment of the 15 included articles using QUADAS-2 is shown in On-line Table 2.

\section{Pooled Measures of Diagnostic Accuracy and Clinical Utility}

Our results showed that pooled sensitivity, specificity, positive likelihood ratio, negative likelihood ratio, and diagnostic odds ratio were 0.85 (95\% CI, 0.79-0.89), 0.88 (95\% CI, 0.83-0.91), 7.0 (95\% CI, 5.0-9.9), 0.17 (95\% CI, 0.12-0.24), and 40 (95\% CI, 22-73), respectively (Fig 2). The area under the receiver operating characteristic curve was 0.93 (95\% CI, 0.90-0.95), which suggested high diagnostic accuracy (Fig 3). Low heterogeneity was detected in these estimates (overall heterogeneity: $\mathrm{I}^{2}=0 \%$; $95 \%$ CI, $0 \%-100 \%, P=.48$; sensitivity: $\mathrm{I}^{2}=27.0 \%$; $95 \% \mathrm{CI}, 0 \%-$ $72.1 \% ; P=.16$; specificity: $\mathrm{I}^{2}=5.8 \%$; $95 \% \mathrm{CI}, 0 \%-78.1 \% ; P=$ .39) (Fig 2).

To assess the clinical utility of our findings, we estimated the posttest probability of tumor recurrence after applying positive and negative likelihood ratios to contrast-enhancing lesions on MR imaging after radiation therapy with a range of pretest probabilities of tumor recurrence. These data are presented in Fig 4.

\section{Meta-Regression and Subgroup Analysis}

To explore the source of heterogeneity, we conducted univariate meta-regression. Of all the covariates, our results showed that the 


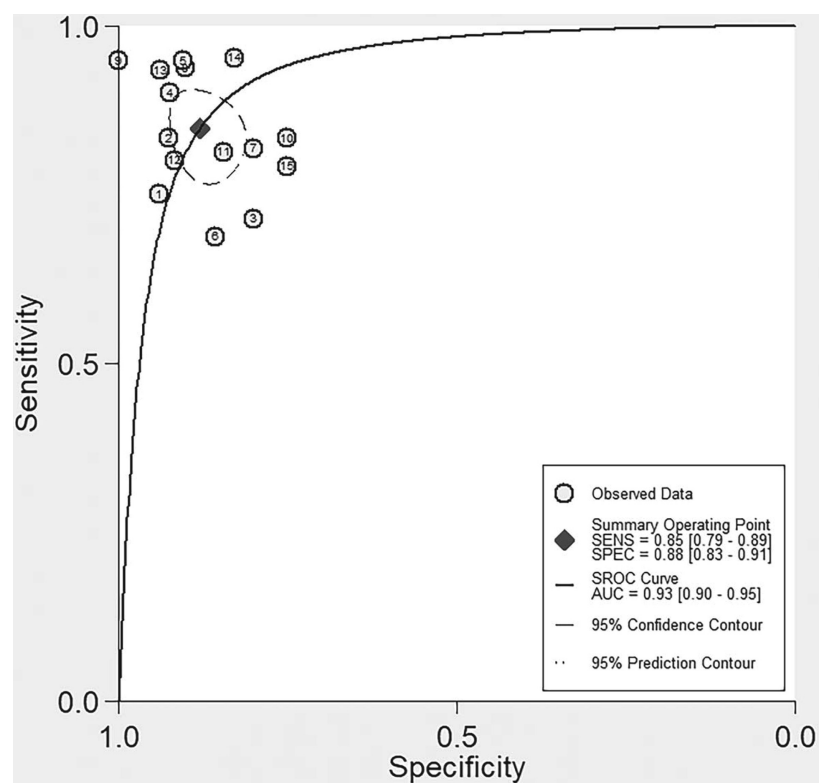

FIG 3. Summary receiver operating characteristic curve of PET. Each circle indicates 1 included study, and the size of the circle indicates the weight of that study. The summary point ( $)$ indicates an estimate of sensitivity and specificity, with the dashed line representing the $95 \%$ confidence region and the dotted line representing the $95 \%$ prediction region.

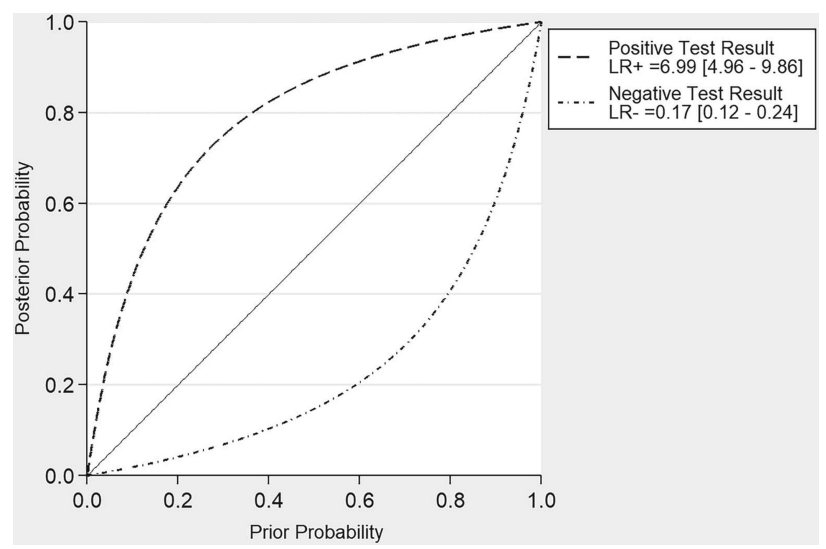

FIG 4. Bayesian plot of estimates of posttest probability of PET after applying pooled positive and negative likelihood ratios to donors with a range of pretest probabilities. $L R+$ indicates pooled positive likelihood ratio; LR-, pooled negative likelihood ratio.

method of quantification by the study (based on lesion versus not, $P=.07$; based on patient versus not, $P=.60$; based on scan versus not, $P=.11)$ contributed to the heterogeneity. Studies analyzed on the basis of lesion ${ }^{8,13,22,23,31-34,37,38,40}$ showed a lower sensitivity $(0.82 ; 95 \% \mathrm{CI}, 0.77-0.87)$ than those based on patient $\mathrm{t}^{24,36}$ (0.91; 95\% CI, $0.78-1.00)$ or PET scan ${ }^{35,39}$ (0.95; 95\% CI, $0.88-$ 1.00). On subgroup analysis of different tracers, 6 studies of ${ }^{18} \mathrm{~F}$ FDG returned a pooled sensitivity of 0.85 (95\% CI, 0.77-0.94) and specificity of 0.90 (95\% CI, $0.83-0.96) ; 5$ studies of ${ }^{18} \mathrm{~F}$-FET returned a pooled sensitivity of $0.83(95 \% \mathrm{CI}, 0.76-0.91)$ and specificity of 0.89 (95\% CI, 0.83-0.95); 2 studies of ${ }^{11} \mathrm{C}-\mathrm{MET}$ returned a pooled sensitivity of $0.86(95 \% \mathrm{CI}, 0.74,0.98)$ and specificity of 0.79 (95\% CI, $0.66-0.93)$; and 2 studies of ${ }^{18} \mathrm{~F}$ FDOPA returned a pooled sensitivity of 0.86 (95\% CI, 0.74-0.97) and specificity of 0.88 (95\% CI, 0.79-0.97). Details are shown in On-line Table 3.

\section{Sensitivity and Publication Bias Analysis}

Sensitivity analysis using the leave-one-out approach demonstrated that the direction and magnitude of estimates were not influenced by any study removed (On-line Table 4). After we excluded the 2 studies that were published as abstracts, ${ }^{24,33}$ the results did not change significantly (On-line Table 4). No publication bias was detected per Deeks funnel plot asymmetry test $(P=.85)($ Fig 5$)$.

\section{DISCUSSION}

The brain is the most common site for central nervous system malignancy. ${ }^{41}$ Brain metastases, which commonly spread from lung, breast, melanoma, colon, and kidney cancers, are at least 10 times more frequent than primary brain tumor. ${ }^{42}$ Radiation therapy can be used therapeutically, prophylactically, or most commonly as palliative (noncurative) treatment for brain metastasis. Radionecrosis, a commonly observed complication that is impossible to eliminate, typically shows delayed occurrence and has been reported to occur in up to $25 \%$ of patients after the completion of radiation therapy. In fact, depending on the irradiated volume and radiation dose, the risk of radionecrosis may be as high as $50 \% .^{12}$ Furthermore, clinical monitoring may also be challenging because both recurrent brain metastasis and radiation injury are characterized by similar neurologic symptoms and MR imaging signs. ${ }^{43}$ Because tumor cells are hypermetabolic (with an increased rate of glycolysis) with elevated hexokinase activity and lower glucose-6-phosphatase activity while radiation necrosis is hypometabolic, ${ }^{44}$ PET using tracers such as ${ }^{18} \mathrm{~F}-\mathrm{FDG},{ }^{11} \mathrm{C}-\mathrm{MET}$, ${ }^{18} \mathrm{~F}$-FET, and ${ }^{18} \mathrm{~F}$-FDOPA has been introduced as a diagnostic tool for differentiation.

In our meta-analysis, we included 15 studies focusing on amino acid and FDG-PET in differentiating brain metastasis recurrence from radionecrosis after radiation therapy. The results showed that PET had an overall pooled sensitivity of 0.85 , specificity of 0.88 , positive likelihood ratio of 7.0, negative likelihood ratio of 0.17 , and diagnostic odds ratio of 40 . A summary receiver operating characteristic curve was developed with an area under the receiver operating characteristic curve of 0.93 . Low heterogeneity was found, and further meta-regression analysis demonstrated that the analytic method used by the individual study may contribute to the heterogeneity. Most studies $8,13,22,23,31-34,37,38,40$ ( $n=11)$ analyzed data based on contrast-enhancing lesions found on brain MR imaging. Only 2 studies $^{24,36}$ analyzed data based on patients; and 2 others, ${ }^{35,39}$ based on each PET scan. Study design, country, radiation therapy methods, tracers, cutoff indexes, sample size, median age, and male percentage did not contribute to heterogeneity. Sensitivity analysis using a leave-oneout approach and publication bias detection showed that our results were stable and reliable. Furthermore, the Bayesian plot demonstrated that PET scans had clinical significance to some extent.

Among our included studies, Guffens et $\mathrm{al}^{33}$ had the lowest diagnostic accuracy of $71.8 \%$ and sensitivity of $68.8 \%$. This may be due to differences in baseline characteristics of the included 


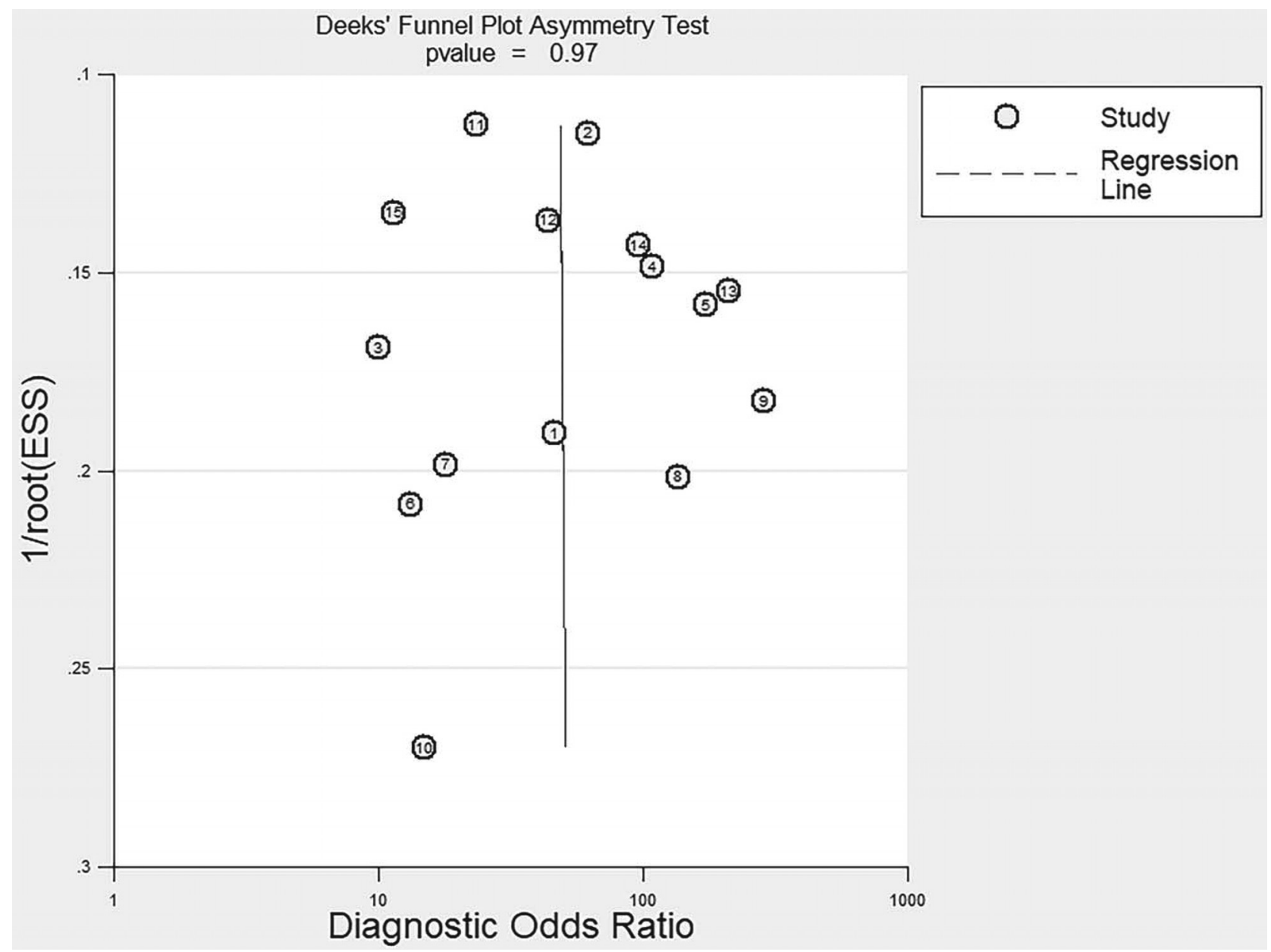

FIG 5. Funnel plot for Deeks' test based on the data of PET for differentiating brain metastasis recurrence from radionecrosis after radiation therapy.

cohort. For example, median age, sex proportion, and radiation therapy method were all unclear in this study. Furthermore, the origin of the brain metastasis was less varied than in other studies. Horky et $\mathrm{al}^{35}$ had a specificity of $100 \%$ with no false-positives. The small sample size (25 patients with 27 lesions) and the brain lesion size cutoff used (only lesions with volume at least $0.51 \mathrm{~cm}^{3}$ were evaluated with FDG-PET) may contribute to this result. The low specificity $(75 \%)$ reported by Lai et $\mathrm{al}^{36}$ may also have been affected by the small sample size.

Meta-regression and subgroup analyses did not find any difference in the cutoff index using quantitative methods with or without the time-activity curve and visual scale. However, we still question whether a threshold effect made a difference because each study used different indexes. Moreover, we did not find any difference in the various tracers used, which is contrary to some previous studies in the literature. ${ }^{8,13,39}$ This could be attributed to the small number of studies in our subgroup analysis. A recent study by Tomura et $\mathrm{al}^{45}$ demonstrated that PET using ${ }^{11} \mathrm{C}-\mathrm{MET}$ was superior to FDG-PET in differentiating radiation necrosis from recurrent tumor after gamma knife radiosurgery. The strength of the study by Tomura et $\mathrm{al}^{45}$ is its direct comparison of FDG-PET and ${ }^{11} \mathrm{C}$-MET PET by performing the 2 on the same patients. However, the study is limited by the small cohort size (15 patients with 18 lesions) as well as testing only 1 amino acid PET tracer. Future larger studies directly comparing the different tracers on the same lesions are needed to prove the superiority of amino acid PET over FDG-PET.

Other imaging methods have also been used to differentiate brain metastasis recurrence from radionecrosis after radiation therapy, but the studies are few, and the results are inconsistent. Some researchers focused on the use of conventional MR imaging. Dequesada et al ${ }^{46}$ showed a good diagnostic accuracy (sensitivity $=80 \%$, specificity $=96 \%$ ) in 32 patients using an MR imaging-based parameter called the "lesion quotient," which was defined as the ratio of the nodule as seen on T2 imaging to the total enhancing area on T1 imaging. However, in a subsequent larger study of 51 patients by Stockham et al, ${ }^{15}$ the lesion quotient was inadequately sensitive (59\%) and specific (41\%) for discriminating tumor progression and radionecrosis. Leeman et $\mathrm{al}^{47} \mathrm{cal}-$ culated the edema/lesion volume ratio on preoperative MR imaging of 49 patients and reported a sensitivity of $63 \%$ and specificity of $85 \%$ in differentiating metastasis recurrence from radionecrosis. Using qualitative analysis of the time-dependent changes in lesion morphology on MR imaging of 31 patients treated with SRS for brain metastases, Wagner et $\mathrm{al}^{48}$ showed that all radiationinduced injuries had a black interior area on the subtraction images for 15 to 55 minutes, whereas all malignant lesions had white components $(P<.001)$. Although these studies based on conven- 
tional MR imaging demonstrated promise, all were based on a small number of patients and were experimental in nature with insufficient evidence for clinical use.

Among our included studies, only 2 studies directly commented on the added value of PET over conventional or advanced MR imaging. Bělohlávek et $\mathrm{al}^{22}$ demonstrated that a subsequent positive FDG-PET finding following a positive conventional MR imaging finding could increase the probability of correctly identifying metastasis recurrence by $68 \%$ (from $32 \%$ to $100 \%$ ) and a negative FDG-PET finding could decrease this probability by $20.9 \%$ (from $32 \%$ to $11.1 \%$ ). Chao et $\mathrm{al}^{31}$ showed a higher sensitivity of FDG-PET when coregistered with MR imaging (FDGPET with MR imaging coregistration versus FDG-PET alone, 86\% versus $65 \%$ ). A recent cost-effectiveness analysis ${ }^{49}$ on the use of FET-PET in addition to MR imaging compared with MR imaging alone for the diagnosis of recurrent brain metastases demonstrated that the additional use of FET-PET increased the rate of a correct diagnosis by $42 \%$ compared with MR imaging alone and suggests that it may be cost-effective. However, with the relatively low spatial resolution of PET and variable uptake of metastasis from tumors of different origins, the sensitivity of PET would not be expected to reach $100 \%$. The specificity can also hardly reach $100 \%$ because of high background activity. Thus, the clinical application may be somewhat limited. Although we could show that amino acid or FDG-PET had high sensitivity and specificity in distinguishing radiation necrosis from tumor recurrence, the significant costs associated with the use of PET may not justify its use in the patient population with brain metastasis. To demonstrate its cost-effectiveness, future studies should set carefully selected inclusion criteria and attempt to link the diagnostic utility of PET in the selected cohort to changes in management or improvement in survival. ${ }^{50}$ Potential cost savings include avoiding biopsies and futile treatment when diagnosis is uncertain.

In addition to ${ }^{18} \mathrm{~F}-\mathrm{FDG},{ }^{11} \mathrm{C}-\mathrm{MET},{ }^{18} \mathrm{~F}$-FET, and ${ }^{18} \mathrm{~F}$-FDOPA included in our analysis, other PET tracers such as ${ }^{18} \mathrm{~F}$-fluorocholine, ${ }^{51}{ }^{18} \mathrm{~F}$-fluorothymidine $\left({ }^{18} \mathrm{~F}\right.$-FLT $),{ }^{52,53}$ and ${ }^{11} \mathrm{C}$-choline $\mathrm{e}^{54,55}$ can also be used in differentiating tumor recurrence and radiation necrosis. For example, Enslow et al $^{52}$ compared FLT-PET with FDG-PET in differentiating radiation necrosis from recurrent glioma and demonstrated a higher diagnostic accuracy of FDG-PET (area under the receiver operating characteristic curve: FLT-PET versus FDG-PET = mean, 0.86; 95\% CI, 0.67-1.00, versus mean, $0.93 ; 95 \%$ CI, $0.80-1.00)$. However, the study focused on primary gliomas and was limited by a small cohort size $(n=15)$.

Other studies in the literature focused on the use of advanced imaging techniques to distinguish brain metastasis recurrence from radionecrosis after radiation therapy. In a study of 14 patients with cerebral metastasis who had clinical or radiographic progression following SRS, Lai et $\mathrm{al}^{36}$ reported the sensitivity and specificity of thallium SPECT to be only $50.0 \%$ and $62.5 \%$, respectively. In the same study, arterial spin-labeling MR imaging had a sensitivity of $83.3 \%$ and specificity of $100.0 \%$. Cicone et $\mathrm{al}^{23}$ reported that the overall accuracy of MRP was lower than that of ${ }^{18}$ F-FDOPA PET (perfusion-weighted MR imaging versus PET: sensitivity, $86.7 \%$ versus $93.3 \%$; specificity, $68.2 \%$ versus $90.9 \%$; accuracy, $75.6 \%$ versus $91.9 \%$ ) in 42 patients with 50 brain metastases, while Hatzoglou et $\mathrm{al}^{34}$ reported a higher sensitivity of perfusion-weighted MR imaging than ${ }^{18} \mathrm{~F}-\mathrm{FDG}$-PET (91\% versus $82 \%$ ) in 53 patients. However, in the study by Hatzoglou et al, ${ }^{34}$ 29 patients received postoperative partial brain radiation therapy for gliomas and the study cohort was too small for subgroup analysis. Additionally, MR spectroscopy ${ }^{56}$ (sensitivity, $36 \%$; specificity, 55\%), perfusion $\mathrm{CT}^{57}$ (sensitivity, $85.7 \%$; specificity, 100\%), and chemical exchange saturation transfer ${ }^{58}$ were also tested for differential diagnosis in a small number of patients with variable conclusions. Overall, there are insufficient data for analysis and comparison with PET.

To our knowledge, this meta-analysis is the first to assess the diagnostic accuracy of amino acid and FDG-PET in differentiating brain metastasis recurrence from radionecrosis after radiation therapy. There are other meta-analyses focusing on PET in differentiating radionecrosis from recurrence in gliomas and demonstrating good diagnostic value. ${ }^{59-61}$ However, the mechanism of tracer uptake can be different between primary brain tumor and metastasis recurrence because metastasis has vessels that resemble origin tissues, resulting in the lack of any discernible blood-brain barrier, while glioma vessels usually have some remaining bloodbrain barrier structure. ${ }^{62}$

Moreover, we acknowledge several limitations of our study. First, our total pooled sample size was still quite small. Second, we included many retrospective studies $(n=9)$, which reduced the quality of our study. Third, we did not include studies that investigated the diagnostic accuracy of amino acid or FDG-PET in distinguishing postsurgical changes from tumor recurrence. Fourth, further analysis based on cutoff indexes could not be performed due to the lack of studies and incomplete reporting. Finally, there is evidence that ${ }^{18} \mathrm{~F}-\mathrm{FDG}$ can accumulate in abscesses and inflammatory lesions, which can increase false-positive rates and add some bias. ${ }^{63}$

\section{CONCLUSIONS}

In this meta-analysis, we demonstrated that amino acid and FDGPET had good diagnostic accuracy in differentiating brain metastasis recurrence from radionecrosis after radiation therapy. While amino acid and FDG-PET may not be the most effective method to differentiate recurrence from radionecrosis after radiation therapy, it is still superior to other methods that are currently in practice.

\section{APPENDIX \\ PubMed $(\mathbf{n}=106)$}

(Positron-Emission Tomography[MH] OR (("Positron Emission" [TIAB] OR "Positron-Emission"[TIAB]) AND tomography[TIAB]) OR PET[TIAB]) AND (radionecrosis[TIAB] OR "radiation necrosis" [TIAB] OR "radiation-induced necrosis" [TIAB] OR “posttreatment necrosis”[TIAB] OR "radiation injury" [TIAB] OR "radionecrotic"[TIAB] OR "Post-Radiation therapy Necrosis"[TIAB]) AND (recurrence[MH] OR recurrence $^{\star}[\mathrm{TIAB}]$ OR recurrent[TIAB] OR relapse ${ }^{\star}$ [TIAB] OR recrudescence ${ }^{\star}[\mathrm{TIAB}]$ OR neoplasm metastasis[MH] OR metastas $^{\star}[\mathrm{TIAB}]$ OR progression[TIAB]) AND (radiosurgery $[\mathrm{MH}]$ OR radiosurger* [TIAB] OR radiation therapy[MH] OR radiotherap ${ }^{\star}[\mathrm{TIAB}]$ OR "radiation therap ${ }^{\star}$ [TIAB] OR "radiation treatment" [TIAB]) 


\section{Cochrane Library $(\mathrm{n}=6)$}

([mh "Positron-Emission Tomography"] OR (("Positron Emission":ti,ab OR "Positron-Emission":ti,ab) AND tomography: ti,ab) OR PET:ti,ab) AND (radionecrosis:ti,ab OR "radiation necrosis":ti,ab OR “radiation-induced necrosis":ti,ab OR "posttreatment necrosis":ti,ab OR "radiation injury":ti,ab OR "radionecrotic": ti,ab OR "Post-Radiation therapy Necrosis":ti,ab) AND ([mh recurrence] OR recurrence*:ti,ab OR recurrent:ti,ab OR relapse*:ti,ab OR recrudescence*:ti,ab OR [mh "neoplasm metastasis"] OR metastas*: ti,ab OR progression:ti,ab) AND ([mh radiosurgery] OR radiosurger $^{\star}: \mathrm{ti}, \mathrm{ab}$ OR [mh radiation therapy] OR radiotherap*:ti,ab OR "radiation therap ${ }^{\star}$ ":ti,ab OR "radiation treatment":ti,ab)

\section{Embase ( $\mathbf{n}=185)$}

'positron emission tomography'/exp/mj OR ('positron emission': ti,ab OR 'positron-emission':ti,ab AND tomography:ti,ab) OR pet:ti,ab AND (radionecrosis:ti,ab OR 'radiation necrosis':ti,ab OR 'radiation-induced necrosis':ti,ab OR 'posttreatment necrosis':ti,ab OR 'radiation injury':ti,ab OR 'radionecrotic':ti,ab OR 'postradiotherapy necrosis':ti,ab) AND ('cancer recurrence'/ exp/mj OR 'tumor recurrence'/exp/mj OR recurrence*:ti,ab OR recurrent:ti,ab OR relapse ${ }^{\star}:$ ti,ab OR recrudescence* ${ }^{\star}: t i, a b$ OR 'metastasis'/exp/mj OR metastas*:ti,ab OR progression:ti,ab) AND ('radiosurgery'/exp/mj OR radiosurger*:ti,ab OR 'radiation therapy'/exp/mj OR radiotherap ${ }^{*}: t i, a b$ OR 'radiation therap*': ti,ab OR 'radiation treatment':ti,ab) AND [embase]/lim.

Disclosures: Li Yang—RELATED: Grant: Shenghua Yuying Project of Central South University.

\section{REFERENCES}

1. American Brain Tumor Association. Metastatic brain tumors. http:// www.abta.org/secure/metastatic-brain-tumor.pdf. Accessed May 10, 2017

2. Sperduto PW, Kased N, Roberge D, et al. Summary report on the graded prognostic assessment: an accurate and facile diagnosisspecific tool to estimate survival for patients with brain metastases. J Clin Oncol 2012;30:419-25 CrossRef Medline

3. Bai HX, Zou Y, Lee AM, et al. Diagnostic value and safety of brain biopsy in patients with cryptogenic neurological disease: a systematic review and meta-analysis of $\mathbf{8 3 1}$ cases. Neurosurgery 2015;77: 283-95 CrossRef Medline

4. Wang J, Liu X, Ma F, et al. Role of radiotherapy in the treatment of primary mediastinal large B-cell lymphoma. Oncol Lett 2015;10: 2925-30 CrossRef Medline

5. Kocher M, Soffietti R, Abacioglu U, et al. Adjuvant whole-brain radiotherapy versus observation after radiosurgery or surgical resection of one to three cerebral metastases: results of the EORTC 22952-26001 study. J Clin Oncol 2011;29:134-41 CrossRef Medline

6. Aoyama $\mathrm{H}$, Shirato $\mathrm{H}$, Tago $\mathrm{M}$, et al. Stereotactic radiosurgery plus whole-brain radiation therapy vs stereotactic radiosurgery alone for treatment of brain metastases a randomized controlled trial. JAMA 2006;295:2483-91 CrossRef Medline

7. Andrews DW, Scott CB, Sperduto PW, et al. Whole brain radiation therapy with or without stereotactic radiosurgery boost for patients with one to three brain metastases: phase III results of the RTOG 9508 randomised trial. Lancet 2004;363:1665-72 CrossRef Medline

8. Ceccon G, Lohmann P, Stoffels G, et al. Dynamic O-(2-18F-fluoroethyl)-L-tyrosine positron emission tomography differentiates brain metastasis recurrence from radiation injury after radiotherapy. Neuro Oncol 2017;19:281-88 CrossRef Medline

9. Greene-Schloesser D, Robbins ME, Peiffer AM, et al. Radiation- induced brain injury: a review. Front Oncol 2012;2:73 CrossRef Medline

10. Narloch JL, Farber SH, Sammons S, et al. Biopsy of enlarging lesions after stereotactic radiosurgery for brain metastases frequently reveals radiation necrosis. Neuro Oncol 2017;19:1391-97 CrossRef Medline

11. Chen W. Clinical applications of PET in brain tumors. J Nucl Med 2007;48:1468-81 CrossRef Medline

12. Minniti G, Clarke E, Lanzetta G, et al. Stereotactic radiosurgery for brain metastases: analysis of outcome and risk of brain radionecrosis. Radiat Oncol 2011;6:48 CrossRef Medline

13. Lohmann P, Stoffels G, Ceccon G, et al. Radiation injury vs. recurrent brain metastasis: combining textural feature radiomics analysis and standard parameters may increase 18F-FET PET accuracy without dynamic scans. Eur Radiol 2017;27:2916-27 CrossRef Medline

14. Verma N, Cowperthwaite MC, Burnett MG, et al. Differentiating tumor recurrence from treatment necrosis: a review of neuro-oncologic imaging strategies. Neuro Oncol 2013;15:515-34 CrossRef Medline

15. Stockham AL, Tievsky AL, Koyfman SA, et al. Conventional MRI does not reliably distinguish radiation necrosis from tumor recurrence after stereotactic radiosurgery. J Neurooncol 2012;109:149-58 CrossRef Medline

16. Tan Y, Zhang H, Xiao EH. Perivascular epithelioid cell tumour: dynamic CT, MRI and clinicopathological characteristics-analysis of 32 cases and review of the literature. Clin Radiol 2013;68:555-61 CrossRef Medline

17. Menoux I, Armspach JP, Noël G, et al. Techniques d'imagerie utilisées dans le diagnostic différentiel entre radionécrose cérébrale et rechute tumorale après irradiation en conditions stéréotaxiques de métastases cérébrales: revue de la littérature. Cancer/Radiothérapie 2016;20:837-45

18. Karaosmanoglu AD, Onur MR, Ozmen MN, et al. Magnetic resonance imaging of liver metastasis. Semin Ultrasound CT MR 2016; 37:533-48 CrossRef Medline

19. Zhou H, Vallières M, Bai HX, et al. MRI features predict survival and molecular markers in diffuse lower-grade gliomas. Neuro Oncol 2017;19:862-70 CrossRef Medline

20. McGivney DF, Pierre E, Ma D, et al. SVD compression for magnetic resonance fingerprinting in the time domain. IEEE Trans Med Imaging 2014;33:2311-22 CrossRef Medline

21. Galldiks N, Langen KJ, Pope WB. From the clinician's point of view: what is the status quo of positron emission tomography in patients with brain tumors? Neuro Oncol 2015;17:1434-44 CrossRef Medline

22. Bělohlávek O, Šimonová G, Kantorová I, et al. Brain metastases after stereotactic radiosurgery using the Leksell gamma knife: can FDG PET help to differentiate radionecrosis from tumour progression? Eur J Nucl Med Mol Imaging 2003;30:96-100 CrossRef Medline

23. Cicone F, Minniti G, Romano A, et al. Accuracy of F-DOPA PET and perfusion-MRI for differentiating radionecrotic from progressive brain metastases after radiosurgery. Eur J Nucl Med Mol Imaging 2015;42:103-11 CrossRef Medline

24. Heesters M, van der Weide HL, Bolt RA, et al. C-11-Methionine PET and radiotherapy dose distribution in the differentiation of radionecrosis and tumour progression after radiosurgery of brain metastases. Neuro Oncol 2012;14:i74

25. Whiting PF. QUADAS-2: a revised tool for the quality assessment of diagnostic accuracy studies. Ann Intern Med 2011;155:529-36 CrossRef Medline

26. Reitsma JB, Glas AS, Rutjes AW, et al. Bivariate analysis of sensitivity and specificity produces informative summary measures in diagnostic reviews. J Clin Epidemiol 2005;58:982-90 Medline

27. Swets JA. Measuring the accuracy of diagnostic systems. Science 1988;240:1285-93 Medline

28. Higgins JP, Thompson SG, Deeks JJ, et al. Measuring inconsistency in meta-analyses. BMJ 2003;327:557-60 Medline

29. Anzures-Cabrera J, Higgins JP. Graphical displays for meta-analysis: an 
overview with suggestions for practice. Res Synth Methods 2010;1: 66-80 CrossRef Medline

30. Sutton AJ, Abrams KR. Bayesian methods in meta-analysis and evidence synthesis. Stat Methods Med Res 2001;10:277-303 CrossRef Medline

31. Chao ST, Suh JH, Raja S, et al. The sensitivity and specificity of FDG PET in distinguishing recurrent brain tumor from radionecrosis in patients treated with stereotactic radiosurgery. Int J Cancer 2001;96: 191-97 Medline

32. Galldiks N, Stoffels G, Filss CP, et al. Role of O-(2-18F-fluoroethyl)L-tyrosine PET for differentiation of local recurrent brain metastasis from radiation necrosis. J Nucl Med 2012;53:1367-74 CrossRef Medline

33. Guffens F, Maes A, Van Laere K, et al. Dynamic O-(2-18F-fluoroethyl)-l-tyrosine PET improves differentiation of local recurrent brain metastasis from radiation necrosis. J Nucl Med 2015;56(suppl 3):628

34. Hatzoglou V, Yang TJ, Omuro A, et al. A prospective trial of dynamic contrast-enhanced MRI perfusion and fluorine-18 FDG PET-CT in differentiating brain tumor progression from radiation injury after cranial irradiation. Neuro Oncol 2016;18:873-80 CrossRef Medline

35. Horky LL, Hsiao EM, Weiss SE, et al. Dual phase FDG-PET imaging of brain metastases provides superior assessment of recurrence versus post-treatment necrosis. J Neurooncol 2011;103:137-46 CrossRef Medline

36. Lai G, Mahadevan A, Hackney D, et al. Diagnostic accuracy of PET, SPECT, and arterial spin-labeling in differentiating tumor recurrence from necrosis in cerebral metastasis after stereotactic radiosurgery. AJNR Am J Neuroradiol 2015;36:2250-55 CrossRef Medline

37. Lizarraga KJ, Allen-Auerbach M, Czernin J, et al. 18F-FDOPA PET for differentiating recurrent or progressive brain metastatic tumors from late or delayed radiation injury after radiation treatment. J Nucl Med 2014;55:30-36 CrossRef Medline

38. Matuszak J, Waissi W, Clavier JB, et al. Métastases cérébrales: apport de l'acquisition tardive en TEP/TDM au 18F-FDG pour le diagnostic différentiel entre récurrence tumorale et radionécrose. Médecine Nucléaire 2016;40:129-41

39. Romagna A, Unterrainer M, Schmid-Tannwald C, et al. Suspected recurrence of brain metastases after focused high dose radiotherapy: can [18F]FET- PET overcome diagnostic uncertainties? Radiat Oncol 2016; 11:139 CrossRef Medline

40. Terakawa Y, Tsuyuguchi N, Iwai Y, et al. Diagnostic accuracy of 11C-methionine PET for differentiation of recurrent brain tumors from radiation necrosis after radiotherapy. J Nucl Med 2008;49: 694-99 Medline

41. Lei D, Li K, Li L, et al. Disrupted functional brain connectome in patients with posttraumatic stress disorder. Radiology 2015;276: 818-27 CrossRef Medline

42. Vargo MM. Brain tumors and metastases. Phys Med Rehabil Clin N Am 2017;28:115-41 CrossRef Medline

43. Chao ST, Ahluwalia MS, Barnett GH, et al. Challenges with the diagnosis and treatment of cerebral radiation necrosis. Int J Radiat Oncol Biol Phys 2013;87:449-57 CrossRef Medline

44. Gao S, Li S, Yang X, et al. 18FDG PET-CT for distant metastases in patients with recurrent head and neck cancer after definitive treatment: a meta-analysis. Oral Oncol 2014;50:163-67 CrossRef Medline

45. Tomura N, Kokubun M, Saginoya T, et al. Differentiation between treatment-induced necrosis and recurrent tumors in patients with metastatic brain tumors: comparison among 11C-methionine-PET, FDG-PET, MR permeability imaging, and MRI-ADC_-preliminary results. AJNR Am J Neuroradiol 2017;38:1520-27 CrossRef Medline

46. Dequesada IM, Quisling RG, Yachnis A, et al. Can standard magnetic resonance imaging reliably distinguish recurrent tumor from radiation necrosis after radiosurgery for brain metastases? A radiographic-pathological study. Neurosurgery 2008;63:898-903; discussion 904 CrossRef Medline

47. Leeman JE, Clump DA, Flickinger JC, et al. Extent of perilesional edema differentiates radionecrosis from tumor recurrence follow- ing stereotactic radiosurgery for brain metastases. Neuro Oncol 2013;15:1732-38 CrossRef Medline

48. Wagner S, Lanfermann H, Eichner G, et al. Radiation injury versus malignancy after stereotactic radiosurgery for brain metastases: impact of time-dependent changes in lesion morphology on MRI. Neuro Oncol 2017;19:586-94 CrossRef Medline

49. Heinzel A, Müller D, Yekta-Michael SS, et al. O-(2-18F-fluoroethyl)L-tyrosine PET for evaluation of brain metastasis recurrence after radiotherapy: an effectiveness and cost-effectiveness analysis. Neuro Oncol 2017;19:1271-78 CrossRef Medline

50. Miles K, McQueen L, Ngai S, et al. Evidence-based medicine and clinical fluorodeoxyglucose PET/MRI in oncology. Cancer Imaging 2015;15:18 CrossRef Medline

51. Spaeth N, Wyss MT, Weber B, et al. Uptake of 18F-fluorocholine, 18F-fluoroethyl-L-tyrosine, and 18F-FDG in acute cerebral radiation injury in the rat: implications for separation of radiation necrosis from tumor recurrence. J Nucl Med 2004;45:1931-38 Medline

52. Enslow MS, Zollinger LV, Morton KA, et al. Comparison of $18 \mathrm{~F}-$ fluorodeoxyglucose and 18F-fluorothymidine PET in differentiating radiation necrosis from recurrent glioma. Clin Nucl Med 2012; 37:854-61 CrossRef Medline

53. Spence AM, Muzi M, Link JM, et al. NCI-sponsored trial for the evaluation of safety and preliminary efficacy of $3^{\prime}$-deoxy-3'$[18 \mathrm{~F}]$ fluorothymidine (FLT) as a marker of proliferation in patients with recurrent gliomas: preliminary efficacy studies. Mol Imaging Biol 2009;11:343-55 CrossRef Medline

54. Takenaka S, Asano Y, Shinoda J, et al. Comparison of (11)C-methionine, (11)C-choline, and (18)F-fluorodeoxyglucose-PET for distinguishing glioma recurrence from radiation necrosis. Neurol Med Chir (Tokyo) 2014;54:280-89 CrossRef Medline

55. Tan H, Chen L, Guan Y, et al. Comparison of MRI, F-18 FDG, and 11C-choline PET/CT for their potentials in differentiating brain tumor recurrence from brain tumor necrosis following radiotherapy. Clin Nucl Med 2011;36:978-81 CrossRef Medline

56. Huang J, Wang AM, Shetty A, et al. Differentiation between intraaxial metastatic tumor progression and radiation injury following fractionated radiation therapy or stereotactic radiosurgery using MR spectroscopy, perfusion MR imaging or volume progression modeling. Magn Reson Imaging 2011;29:993-1001 CrossRef Medline

57. Vidiri A, Guerrisi A, Pinzi V, et al. Perfusion computed tomography (PCT) adopting different perfusion metrics: recurrence of brain metastasis or radiation necrosis? Eur J Radiol 2012;81:1246-52 CrossRef Medline

58. Mehrabian H, Desmond KL, Soliman H, et al. Differentiation between radiation necrosis and tumor progression using chemical exchange saturation transfer. Clin Cancer Res 2017;23:3667-75 CrossRef Medline

59. Deng SM, Zhang B, Wu YW, et al. Detection of glioma recurrence by ${ }^{11} \mathrm{C}$-methionine positron emission tomography and dynamic susceptibility contrast-enhanced magnetic resonance imaging: a metaanalysis. Nucl Med Commun 2013;34:758-66 CrossRef Medline

60. Nihashi T, Dahabreh IJ, Terasawa T. Diagnostic accuracy of PET for recurrent glioma diagnosis: a meta-analysis. AJNR Am J Neuroradiol 2013;34:944-50, S1-11 CrossRef Medline

61. Wang $\mathrm{X}, \mathrm{Hu} \mathrm{X}$, Xie $\mathrm{P}$, et al. Comparison of magnetic resonance spectroscopy and positron emission tomography in detection of tumor recurrence in posttreatment of glioma: a diagnostic metaanalysis. Asia Pac J Clin Oncol 2015;11:97-105 CrossRef Medline

62. Cha S, Lupo JM, Chen M, et al. Differentiation of glioblastoma multiforme and single brain metastasis by peak height and percentage of signal intensity recovery derived from dynamic susceptibilityweighted contrast-enhanced perfusion MR imaging. AJNR Am J Neuroradiol 2007;28:1078-84 Medline

63. Antinori A, De Rossi G, Ammassari A, et al. Value of combined approach with thallium-201 single-photon emission computed tomography and Epstein-Barr Virus DNA polymerase chain reaction in CSF for the diagnosis of AIDS-related primary CNS lymphoma. J Clin Oncol 1999;17:554-60 Medline 